\title{
Social Computers for the Social Animal: State-of-the-Art and Future Perspectives of Social Signal Processing
}

\author{
Alessandro Vinciarelli \\ IDIAP Research Institute \\ Switzerland
}

\begin{abstract}
Following Aristotle, "Man is by nature a social animal; an individual who is unsocial naturally and not accidentally is either beneath our notice or more than human." This is more than an abstract philosophical statement if, twenty five centuries after, we observe that people have exactly the same social behavior whether they interact with a computer or with another person. Furthermore, there is evidence that users tend to appreciate more computers displaying social behaviors similar to those they appreciate in people. This body of evidence suggests that there is a gap between current, unsocial, computers and user expectations for social behavior.

Social Signal Processing (SSP) is the new, emerging, domain that aims at making computers as social as their human users by modeling people and groups involved in social interactions. The SSP approach focuses on analysis, understanding and synthesis of social signals, the complex aggregates of nonverbal behavioral cues through which people convey their attitude towards others (including machines) and social environments. The development of SSP technologies will help computers to adapt to users like people adapt to others (i.e. depending on the kind of interaction and social context), and to personalize their interface in terms of characteristics socially desirable for the users.
\end{abstract}

